\title{
9
}

Subdirección General de Inspección, Certificación y Asistencia Técnica

de Comercio Exterior*

\section{CONVENIO CITES: INSTRUMENTO DE POLÍTICA COMERCIAL REGULADOR DEL COMERCIO DE VIDA SILVESTRE}

Durante los años sesenta, debido al excesivo uso comercial de los recursos naturales, muchos Gobiernos, y también la sociedad, empiezan a tomar conciencia de la necesidad de adoptar medidas que permitan equilibrar el uso sostenible de los recursos naturales y la conservación de los mismos. Ello llevó a que 21 países firmaran en el año 1973 el Convenio sobre el Comercio Internacional de Especies Amenazadas de Fauna y Flora Silvestres (CITES), convenio al cual paulatinamente se han ido adhiriendo otros países, hasta un total de 183 . Hoy se puede afirmar que CITES es un acuerdo multilateral ambiental que ha conseguido combinar de manera eficaz la relación comercio y medio ambiente. Sin embargo, el rápido incremento en los últimos años del tráfico ilegal de vida silvestre asociado a redes organizadas de delincuencia internacional está impulsando la adopción de medidas excepcionales entre los Gobiernos para reforzar la aplicación de CITES y combatir enérgicamente el comercio ilegal.

Palabras clave: uso sostenible, desarrollo sostenible, especies, tráfico.

Clasificación JEL: F18, F13, Q56, Q57, Q58.

\section{Introducción}

Desde el inicio de la era humana, tradicionalmente, el hombre ha utilizado los recursos naturales y los productos de la vida silvestre como medio de subsistencia. Es en la Edad Contemporánea, y especialmente tras la Revolución Industrial, cuando asistimos a una sobreexplotación de los recursos naturales que

\footnotetext{
*Este artículo ha sido elaborado por: Mercedes Núñez Román Coordinadora Nacional CITES; Marta Alzás de la Fuente, Jefe de Área CITES, y Alicia Sánchez Muñoz, Subdirectora General de Inspección, Certificación y Asistencia Técnica de Comercio Exterior.

Versión de agosto de 2017.
}

lleva a muchos países a adoptar medidas al respecto para reconducir la situación. Así, en 1933 se firma la Convención sobre la Fauna y la Flora, primer acuerdo multilateral en el que se mencionó la interacción entre comercio y medio ambiente. Sin embargo, no es hasta principios de los setenta cuando esta materia empieza a ser analizada con un alto interés por parte de la opinión pública.

Los antecedentes del Convenio sobre el Comercio Internacional de Especies Amenazadas de Fauna y Flora Silvestres, conocido como CITES, que es el acrónimo del nombre en inglés (Convention on $\triangleright$ 
International Trade in Endangered Species of Wild Fauna and Flora), se remontan a 1962, cuando la UICN (Unión Internacional para la Conservación de la Naturaleza), consciente del intensivo uso de los recursos naturales, señala la necesidad de establecer un control del comercio internacional de especies de fauna y flora, ya que éste había sido identificado como uno de los factores de extinción de especies y de pérdida de biodiversidad. Posteriormente, en 1972, tiene lugar en Estocolmo la Conferencia Mundial sobre Medio Ambiente, que acuerda elaborar un tratado internacional regulador del comercio de especies amenazadas. En 1973 se reúne en Washington la primera conferencia plenipotenciaria que redacta y aprueba el texto. El 3 de marzo de 1973 veintiún países firman el convenio, el cual entra en vigor el 1 de julio de 1975. Actualmente, el número de partes firmantes es ya de 183, incluyendo a la Unión Europea, tras la aprobación en 2013 de la segunda enmienda del convenio, conocida como la enmienda de Gaborone.

Desde el inicio de la propia actividad comercial ha existido una estrecha relación entre el comercio internacional y el medio ambiente, pero no es hasta la década de los noventa cuando los países empiezan a tomar conciencia de la indudable influencia que tiene la actividad comercial internacional sobre la conservación de los medios naturales. A partir de la Conferencia de las Naciones Unidas sobre Comercio y Desarrollo (CNUCED), celebrada en Río de Janeiro en 1992, los instrumentos jurídicos referidos al medio ambiente se han multiplicado considerablemente.

Las cuestiones medioambientales que tienen una relación directa con el comercio (recogidas en el artículo xx g) del Acuerdo General sobre Aranceles Aduaneros y Comercio, GATT) ocupan poco a poco un lugar más destacado en el panorama internacional debido a su estrecha relación con los aspectos económicos y a la necesidad de integrar políticas medioambientales y políticas económicas para conseguir un futuro sostenible en cualquier parte del mundo.

\section{El Convenio CITES}

\subsection{Aspectos generales}

CITES es un acuerdo multilateral concertado entre Gobiernos (partes) cuya finalidad es velar para que el comercio internacional de especímenes de animales y plantas silvestres no constituya una amenaza para su supervivencia. Se enmarca dentro de los acuerdos multilaterales de protección medioambiental y es, sin duda, el que mayor representatividad ha adquirido en este ámbito (PNUMA, 2005).

El preámbulo del CITES recoge los principios constitutivos del mismo, que son los de cooperación internacional, soberanía, valoración y equidad intergeneracional.

La misión del CITES es conservar la diversidad biológica y contribuir a su utilización sostenible velando por que ninguna especie de fauna y flora silvestres se siga sometiendo a una explotación insostenible por el comercio internacional, contribuyendo así a una reducción considerable del índice de pérdida de biodiversidad (CITES, 2013). El principio en el que se fundamenta el convenio no es la conservación a ultranza, sino la utilización sostenible que permita la conservación de la biodiversidad. En línea con este principio, CITES reconoce el legítimo derecho de los países a utilizar sus recursos de manera sostenible.

El espíritu de combinar conservación y desarrollo queda reflejado en dos resoluciones adoptadas por la Conferencia de las Partes $\triangleright$ 
(CdP) sobre cómo el comercio puede favorecer la conservación de la fauna y flora silvestres y el desarrollo de las comunidades locales (Resolución conf. 8.3 [Rev. CoP. 13], CITES, 1992) y sobre cómo el intercambio comercial es beneficioso para la conservación de especies y ecosistemas (Resolución Conf. 10.13 [Rev. CoP15], CITES, 1997). Puede decirse que CITES ha conseguido llegar a un equilibrio entre comercio y conservación gracias a una eficaz regulación del comercio internacional.

CITES funciona como un instrumento de política comercial internacional cuya aplicación se lleva a cabo en el ámbito nacional mediante un sistema de concesión de permisos y certificados, y un régimen de control. De acuerdo con el artículo Ix del convenio, cada país parte tiene la obligación, entre otras muchas funciones, de designar una o más autoridades administrativas responsables, y de la concesión y emisión de los citados permisos y certificados. En el caso de España hay nombradas dos autoridades administrativas, siendo la principal la Dirección General de Política Comercial y Competitividad y la adicional, el Departamento de Aduanas e Impuestos Especiales de la Agencia Estatal de la Administración Tributaria (AEAT). Igualmente, cada país parte debe designar una o más autoridades científicas encargadas de asesorar respecto al efecto del comercio sobre la situación de las especies. En el caso de España, esta autoridad corresponde a la Dirección General de Calidad, Evaluación Ambiental y Medio Natural, perteneciente al Ministerio de Agricultura y Pesca, Alimentación y Medio Ambiente.

Las especies amparadas por el CITES están incluidas en tres apéndices denominados I, II y III en función del grado de protección y el nivel de comercio internacional al que están sometidas. El Apéndice I incluye las especies que se encuentran en peligro de extinción y, por tanto, están sometidas a un mayor nivel de protección y restricción comercial. El Apéndice II incluye especies que no se encuentran necesariamente en peligro de extinción pero cuyo comercio debe controlarse a fin de evitar una utilización incompatible con su supervivencia. El Apéndice III incluye especies que están protegidas al menos en un país, el cual ha solicitado la asistencia de otras partes del CITES para controlar su comercio. El número de especies reguladas por CITES ha aumentado considerablemente y actualmente alrededor de unas 5.000 especies de animales y cerca de $\mathbf{3 0 . 0 0 0}$ especies de plantas se encuentran amparadas por el CITES (Tabla 1).

TABLA 1

NÚMERO DE ESPECIES INCLUIDAS EN LOS APÉNDICES DE CITES

\begin{tabular}{|l|c|c|c|}
\hline \multicolumn{1}{|c|}{ Fauna } & Apéndice I & Apéndice II & Apéndice III \\
\hline Mamíferos & 307 & 319 & 53 \\
Aves & 165 & 1.275 & 35 \\
Reptiles & 86 & 535 & 55 \\
Anfibios & 16 & 98 & - \\
Peces & 15 & 71 & - \\
Invertebrados & 66 & 2.101 & 17 \\
Total fauna & $\mathbf{6 5 5}$ & $\mathbf{4 . 3 9 9}$ & $\mathbf{1 6 0}$ \\
Total flora & $\mathbf{2 9 8}$ & $\mathbf{2 8 . 6 7 9}$ & $\mathbf{1 0}$ \\
Total & $\mathbf{9 5 3}$ & $\mathbf{3 3 . 0 7 8}$ & $\mathbf{1 7 1}$ \\
\hline Fuente: Secretaría CITES (www.cites.org). \\
\hline \multicolumn{4}{|l}{} \\
\hline
\end{tabular}

Es importante puntualizar que en los apéndices de CITES se incluyen especies, pero los productos que se comercializan son especímenes de esas especies que comprenden no sólo los ejemplares vivos o muertos, sino también sus partes y derivados, siendo estos los productos que abarcan el mayor volumen del comercio, afectando a importantes sectores económicos. La casuística de partes y derivados es muy amplia y a modo de ejemplo se pueden citar, entre otros: manufacturas de piel (bolsos, zapatos, $\square$ 
cinturones), instrumentos musicales, trofeos de caza, colmillos y figuras de marfil, preparados farmacéuticos, cosméticos, etcétera.

Las características de las especies incluidas en cada apéndice, así como los requisitos necesarios para su comercio internacional, se encuentran recogidos en el propio texto del convenio, estableciendo los mecanismos de regulación de la actividad comercial. Este carácter operativo del convenio, además de facilitar su aplicación, ha influido en la gran eficacia del mismo. Cabe destacar que desde su entrada en vigor no se ha extinguido ninguna especie amparada por CITES como resultado de su comercio.

Actualmente se desarrolla la Visión Estratégica CITES 2008-2020, que consta de dos propósitos básicos: mejorar el funcionamiento de la convención y garantizar que las políticas CITES se apoyen mutuamente; así como garantizar que los apéndices reflejen debidamente las necesidades de las especies, basando sus decisiones en información científica sólida.

\subsection{EI Convenio CITES como instrumento de política comercial}

CITES actúa como una poderosa herramienta de regulación comercial para las partes, de la mano de los principios de regulación comercial establecidas en su más alto nivel por la Organización Mundial del Comercio. Las disposiciones sobre comercio establecidas por CITES promueven la apertura, la no discriminación, la transparencia y la previsibilidad de las relaciones comerciales mundiales y, al mismo tiempo, permiten que los Gobiernos emprendan acciones encaminadas a perseguir objetivos ambientales y otros objetivos cruciales de bienestar social. Hasta la fecha, en ningún procedimiento de solución de diferencias de la OMC se ha impugnado directamente una medida comercial de CITES.

$\mathrm{Al}$ igual que otros tratados e instrumentos comerciales, CITES incluye diferentes mecanismos encaminados a facilitar su aplicación y funcionamiento. Entre ellos, la inclusión de especies en los apéndices, la realización de enmiendas, el establecimiento de exenciones o el establecimiento de cupos. A todos ellos nos referiremos brevemente. Por lo que se refiere a la inclusión de especies en los apéndices, ésta se basa en criterios biológicos y comerciales definidos en la 9. ${ }^{-}$Conferencia de las Partes (Resolución Conf. 9.24 [Rev. CoP15], CITES, 1994). Cualquier inclusión de una especie en un apéndice, o una enmienda del mismo, se acuerda por mayoría de las partes mediante votación. Un caso especial es el del Apéndice III, en el que la inclusión de especies en el mismo, así como las enmiendas, se solicitan unilateralmente, ya que las partes tienen derecho a enmendar e incluir especies en este apéndice para que su comercio sea controlado.

CITES contempla también determinadas exenciones, las cuales se establecieron como principios generales en el momento de la aprobación del convenio. Sobre la base de estas exenciones las partes están autorizadas a comercializar con especímenes pertenecientes a especies en tránsito, especímenes preconvención adquiridos antes de la fecha de su inclusión en los apéndices, bienes personales, del hogar o que formen parte de colecciones o exhibiciones, así como especímenes criados en cautividad, reproducidos artificialmente o destinados a la investigación, para los que generalmente se requiere un permiso o certificado que justifique esta condición.

Otra de las herramientas comerciales de CITES es el establecimiento de un sistema de cupos de exportación, no incluido en el $\triangleright$ 
texto original de la convención, pero desarrollado y aceptado por las partes como un instrumento útil y efectivo para reglamentar el comercio internacional de flora y fauna silvestre en la 14. Reunión de la CdP (Resolución Conf. 14.7 [Rev. CoP15]). Existen diferentes tipos de cupos, como los cupos nacionales o los cupos de exportación compartidos por varias partes, como ocurre con los ejemplares de esturión y de peces espátula, evaluados anualmente para regular el comercio de productos procedentes de stocks de países del área de distribución de estas especies.

Como medida comercial específica del convenio, existe la posibilidad de establecer recomendaciones de suspensión del comercio con Estados parte, cuando se considera que no existen garantías suficientes de un correcto funcionamiento y aplicación de CITES. Esta situación puede darse cuando alguna parte no ha promulgado la necesaria legislación nacional que cubra las disposiciones de CITES, o de forma reiterada no presenta los informes anuales que obligatoriamente deben presentar todas las partes.

Por último, indicar que CITES no permite el comercio con Estados no firmantes del convenio, a menos que estos hayan informado de la competencia de sus autoridades gubernamentales para la emisión de certificados comparables a los dispuestos por la convención. Además, un Estado parte podrá formular una reserva específica en relación a cualquier especie, parte o derivado incluida en los apéndices CITES, para lo que será considerado como Estado no parte en la convención para el comercio de esa especie hasta que retire la reserva formulada.

\subsection{CITES en la Unión Europea}

El texto de CITES establece los requisitos mínimos que las partes se comprometen a aplicar en el momento de su ratificación como «ley de mínimos», pero permite que los países establezcan medidas más estrictas, y este es el caso de la Unión Europea.

La aplicación del Convenio CITES en el seno de la Unión Europea se lleva a cabo de manera uniforme mediante el Reglamento (CE) n. $\circ$ 338/97 del Consejo, de 9 de diciembre de 1996, relativo a la protección de especies de fauna y flora silvestres mediante el control de su

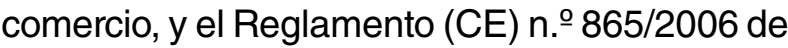
la Comisión, de 4 de mayo de 2006, por el que se establecen disposiciones de aplicación del Reglamento (CE) n.ํ338/97. Dentro de las medidas más estrictas que establece esta normativa se pueden citar, entre otras, las siguientes:

- Mayor grado de protección para algunas especies, por ejemplo, algunos cetáceos, rapaces y especies autóctonas.

- Establecimiento de restricciones de importación para determinadas especies procedentes de países que no pueden asegurar que dichas importaciones vayan en detrimento de la conservación de las mismas.

- Obligatoriedad de marcado para las especies de vertebrados vivos incluidos en el anexo de mayor grado de protección.

La toma de decisiones y gestión del convenio en la Unión Europea se ha dispuesto a través del establecimiento de tres comités: el Comité sobre el Comercio de Fauna y Flora Silvestre de la Comisión, que propone medidas para mejorar la aplicación de los reglamentos comunitarios, y aúna la labor administrativa de los Estados miembros; asimismo, un Grupo de Revisión Científica (SGR), que examina las cuestiones científicas referentes a la aplicación de los reglamentos, y formula $D$ 
recomendaciones en caso de controversia sobre el estatus de conservación de una especie; y por último, un grupo garante de la aplicación, integrado por representantes de cada Estado miembro con responsabilidad en la vigilancia de su cumplimiento, cuya función es examinar todas las cuestiones técnicas relacionadas con la aplicación de los reglamentos y fomentar el intercambio de información.

La Unión Europea es uno de los principales mercados de destino de especímenes de especies incluidas en CITES, destacando el comercio de plantas, seguido del de pieles de reptiles. El Gráfico 1 muestra el comercio de estos productos entre 2012 y 2014.

\subsection{Comercio legal}

Cada día, los siete mil millones de personas que conforman la población mundial consumen biodiversidad, ya se trate de medicamentos, alimentos, ropa, muebles, perfumes, artículos de lujo, etcétera, y, en el mundo globalizado, este consumo crece a un ritmo sin precedentes, lo que hace más necesario establecer medidas que garanticen la sostenibilidad de los recursos.

CITES se sitúa en la intersección entre el comercio, el medio ambiente y el desarrollo. De las especies incluidas en CITES, tan sólo el 3 por 100 están amenazadas de extinción, que corresponden a las especies del Apéndice I, cuyo comercio no está permitido. La mayoría de las especies incluidas en CITES, aproximadamente el 96 por 100, no está necesariamente en peligro de extinción. Son las que se encuentran incluidas en el Apéndice II, cuyo comercio está permitido y genera miles de millones de dólares cada año. Hay muchas formas de comercio legal de especies incluidas en el Apéndice II de CITES, cuyos beneficios para las comunidades locales y países productores son considerables. La regulación del comercio y la aplicación de los requisitos contemplados en CITES ha permitido la recuperación $D$

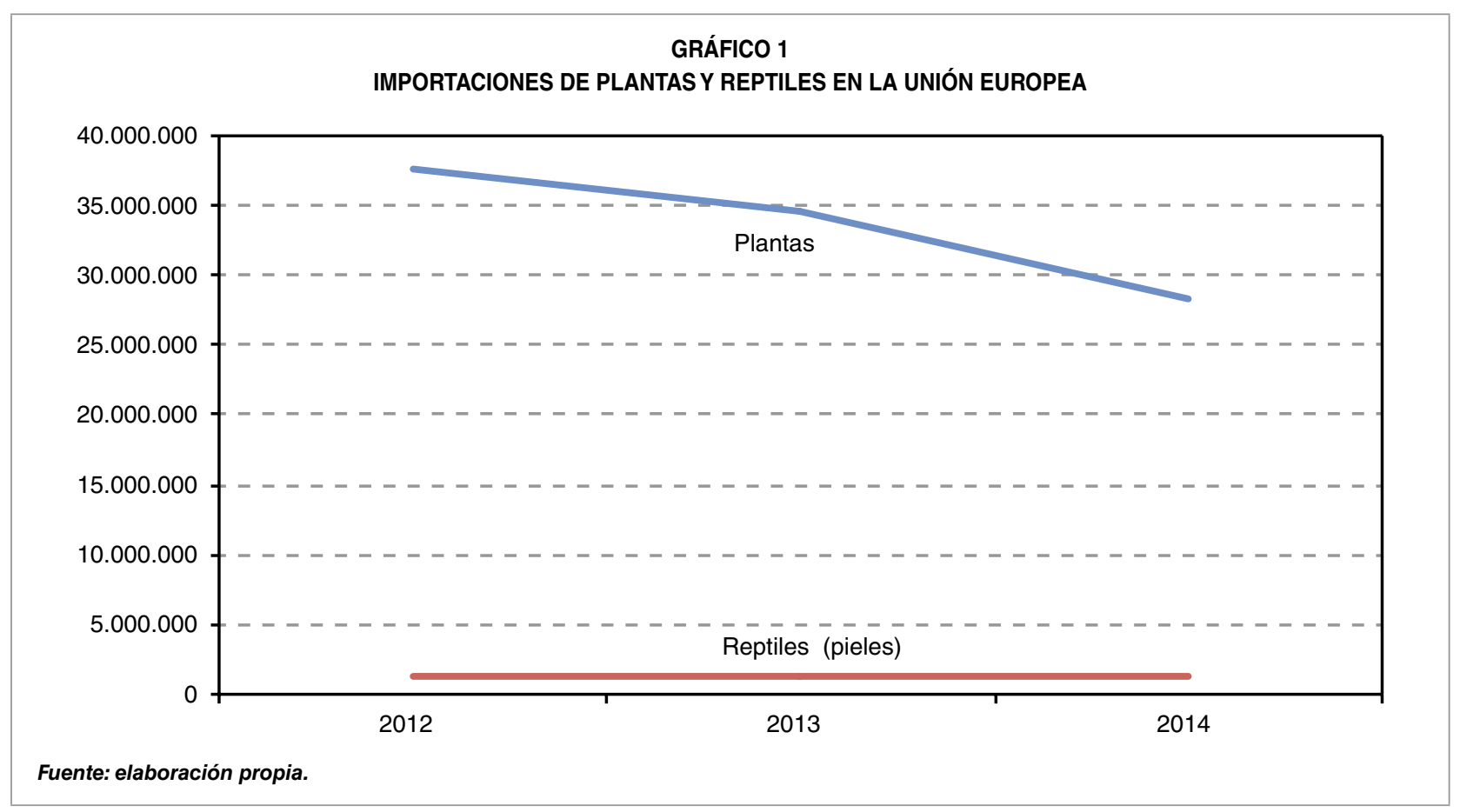


de muchas especies, favoreciendo al mismo tiempo su uso y explotación sostenible. Como ejemplos de utilización sostenible de especies se pueden citar los siguientes: cocodrilos, pitones, vicuñas y ciruelo africano.

\subsubsection{Cocodrilos}

Actualmente se reconocen 26 especies de cocodrilos que se dan en las zonas tropicales y subtropicales. El comercio más importante de estas especies se inició en el siglo XIX cuando la elaboración de productos de piel del cocodrilo americano (Alligator mississippiensis) empezó a cobrar importancia. En los años cincuenta y principios de los sesenta del siglo $x \mathrm{x}$ se estima que se comercializaron anualmente entre 5 y 10 millones de pieles (Inskipp y Wells, 1979). Las poblaciones de cocodrilos disminuyeron drásticamente, en gran medida porque el proceso de la caza estaba eliminando a los segmentos menos sustituibles de la población. En la década de 1970 un elevado porcentaje de las especies del orden Crocodylia estaban en serio peligro de extinción (Jenkins, 1987), principalmente debido a la caza furtiva y al comercio ilegal (Waller y Micucci, 1995).

Sin embargo, la prohibición del aprovechamiento comercial junto con acciones directas de conservación y, posteriormente, la aplicación de planes de manejo basados en metodologías biológicamente sustentables contribuyeron a modificar sustancialmente la situación de las poblaciones silvestres de los cocodrilos (Hutton y Webb, 2002; Ross, 1998). De acuerdo con la UICN (Unión Internacional para la Conservación de la Naturaleza), en la actualidad son cinco las especies que todavía se consideran amenazadas, y en ese sentido es necesario seguir trabajando para garantizar la pervivencia de las mismas.
El comercio internacional actual comprende anualmente 1,5 millones de pieles de cocodrilos, exportadas legalmente de unos treinta países. Sólo en Australia la exportación genera ingresos anuales de unos 16 millones de euros.

\subsubsection{Pitones}

Hay una fuerte demanda de pieles de pitones principalmente de la industria de la moda. Esta demanda ofrece grandes oportunidades para comunidades locales, estimándose que más de medio millón de pieles se exportan anualmente desde el sudeste asiático, generando unos 25 millones de dólares.

El Centro de Comercio Internacional (ITC) ha estimado que, anualmente, se generan mil millones de dólares americanos procedentes de tan sólo cinco especies de pitones incluidas en CITES. Este comercio legal, primordialmente de sus pieles para la industria de la moda, beneficia a los cazadores legales y a las comunidades locales de países de Asia Sudoriental como Indonesia, Malasia y Vietnam, todo ello sin que la sostenibilidad de la especie se vea comprometida.

\subsubsection{Vicuñas}

La vicuña (Vicugna vicugna) es un camélido sudamericano que habita en las zonas andinas, está adaptado a climas extremos, vive entre los 3.500 y $5.000 \mathrm{~m}$ de altura y puede vivir hasta veinte años. Estuvo cerca de su desaparición debido a la caza indiscriminada para la obtención de su pelaje, que se transforma en fibra para la elaboración de prendas. La fibra de la vicuña es bastante más delgada que la cachemira, es muy apreciada y alcanza precios elevados. Estos animales no se reproducen en cautividad, y viven en zonas que no $D$ 
son aptas para la agricultura ni para la ganadería convencional. Por lo tanto, la única forma de garantizar su preservación es proteger las condiciones de su vida silvestre. Gracias a la puesta en marcha de programas de uso sostenible, numerosas comunidades indígenas explotan a las vicuñas. Está prohibido su sacrificio y sólo pueden ser esquiladas cada dos años bajo supervisión estatal.

El comercio de la lana fina de la vicuña ha sido beneficioso para la especie y para las comunidades locales. En los últimos cincuenta años, el número de animales en el medio silvestre ha pasado de 6.000 a 350.000. Su actividad comercial emplea a 900 personas locales, de forma directa o indirecta, tan sólo en Lucanas, un pueblo peruano. Un traje de vicuña puede alcanzar un precio de más de 30.000 euros.

\subsubsection{Ciruelo africano}

La corteza del ciruelo africano (Prunus africana) se utiliza desde hace 35 años en el tratamiento de la hiperplasia prostática benigna y otros trastornos. A pesar de que sólo se utiliza la corteza y de que no es necesaria la tala de árboles, debido a complejos factores ecológicos, sociales y económicos en los principales países exportadores, Camerún, Madagascar, Guinea Ecuatorial y Kenia, la situación de esta especie llegó a ser crítica. Desde 1970, la cosecha de la corteza pasó de ser únicamente de subsistencia a su uso en gran escala en el comercio internacional.

Gracias a un programa conjunto de la Organización Internacional de Maderas Tropicales (OIMT) y CITES, comunidades locales de Camerún que recolectan y comercian corteza seca de ciruelo africano ya no talan los árboles para extraer la corteza y ahora lo cosechan de manera sostenible protegiendo al mismo tiempo los bosques donde crece esta especie. La exportación de la corteza del ciruelo africano es de unas 2.000 toneladas anuales y un valor estimado de 220 millones de dólares.

\subsection{Comercio ilegal}

En detrimento del comercio legal nos encontramos con el comercio ilegal que en los últimos años ha adquirido una relevancia considerable. El tráfico de especies silvestres se ha convertido en uno de los delitos organizados más lucrativos a nivel mundial, colocándose en la tercera posición tras el comercio de drogas y de armas.

Como cualquier otro comercio ilegal, su magnitud es difícil de cuantificar, pero diversas fuentes estiman que alrededor de 350 millones de plantas y animales se venden anualmente en el mercado negro. Los beneficios procedentes de ese tráfico se sitúan, cada año, entre 8.000 y 20.000 millones de euros (UNODOC, CITES). El tráfico afecta a un amplio abanico de especies protegidas, como los elefantes y rinocerontes, los corales, los pangolines, los tigres, los grandes simios y la madera. Como ejemplo, se calcula que el volumen de madera ilegal extraída de nueve países productores de madera tropical fue de más de 80 millones de metros cúbicos en el año 2013.

Especial relevancia ha adquirido en los últimos años la caza ilegal de elefantes y rinocerontes. Desde 2011 se calcula que se han matado entre 20.000 y 30.000 elefantes para la exportación de marfil y, entre 2006 y 2015, más de 5.000 elefantes para el uso de los colmillos en medicina tradicional. El precio pagado por el cuerno de rinoceronte asciende a 40.000 euros el kilo. Cabe destacar, en el caso de España, el incremento en el comercio ilegal de anguila $\square$ 
(Anguilla anguilla) para su exportación a Hong Kong, cuyo precio en el mercado negro alcanza los 1.000 euros el kilo (Comisión Europea, 2014).

El tráfico ilegal de especies silvestres es una de las formas más lucrativas de actividades ilegales en el mundo. Por otra parte, el comercio ilegal de vida silvestre resulta muy atractivo para los delincuentes, ya que, en la mayoría de los países, su represión no es tan prioritaria en comparación con otras formas de tráfico como drogas o armas. Recientemente, además, se dispone de información que confirma la existencia de vínculos con el blanqueo de dinero y otras formas de delincuencia organizada.

Debido al impacto y magnitud que está alcanzando este comercio ilegal, en los últimos años se ha concedido un especial interés a nivel político internacional. En julio de 2015, la Asamblea General de la ONU aprobó la primera resolución sobre este asunto, copatrocinada por todos los Estados miembros de la UE (Naciones Unidas, 2015). Esta cuestión también se ha abordado en otros eventos internacionales importantes, entre los que destacan la Cumbre del G-7 de junio de 2015 y la Cumbre del G-20 de julio de 2017, en la que los líderes aprobaron una declaración sobre cómo combatir la corrupción ligada al comercio ilegal de vida silvestre.

Muchos países están adoptando medidas para combatir el comercio ilegal de vida silvestre. Siguiendo esta línea de actuación, la Unión Europea adoptó en marzo de 2016 un plan de acción contra el tráfico de especies silvestres de amplio alcance para abordar el contrabando de la vida silvestre en la Unión y fortalecer su papel a la hora de combatir las actividades ilegales de forma global, mediante 32 medidas divididas en tres sectores prioritarios: prevención, aplicación y cooperación.

Como medidas adoptadas en relación con la aplicación del plan de acción por parte de la
Unión Europea cabe destacar la prohibición de reexportación de cuernos de rinoceronte desde 2014 y la prohibición de reexportación de marfil en bruto desde julio de este año.

\section{Futuro de CITES}

Es indudable la creciente concienciación que los Gobiernos y la sociedad en general tienen acerca de los problemas ambientales a los que el mundo se enfrenta, y también es indudable la importancia de que los Gobiernos adopten políticas internacionales conjuntas que aseguren la sostenibilidad de nuestros recursos naturales, cuyos problemas y debilidades suelen transcender las fronteras políticas o nacionales. Por ello, CITES ha optado por llevar a cabo una acción concertada a nivel internacional, ofreciendo una oportunidad excepcional para crear sinergias positivas entre los diferentes actores del plano multilateral. Sin duda, la cooperación internacional, la aplicación de medidas sostenibles, la información científica sólida, la integración de la sociedad civil en la toma de decisiones y el apoyo a los países del área de distribución de las especies con mayores volúmenes de comercio constituyen el eje fundamental de la aplicación de CITES.

La 17. - reunión de la Conferencia de las Partes de la Convención celebrada en Johannesburgo, Sudáfrica, en octubre de 2016, consiguió reunir a más de tres mil participantes y abordar más de 120 propuestas relacionadas con la aplicación de CITES, lo que supone una muestra más de que el Convenio CITES es hoy en día un instrumento prioritario para la gestión comercial y de conservación de todos los países.

Se puede afirmar que CITES ha logrado importantes éxitos en lo que a la sostenibilidad de las especies se refiere. Sin embargo, CITES $\triangleright$ 
y los países firmantes deben enfrentarse a un preocupante desafío, el creciente comercio ilegal de especies CITES en todas sus formas, y en particular el denominado ciberdelito, ligado a la proliferación del comercio electrónico ilegal. La voluntad de encontrar soluciones ha llevado a la creación de entidades de apoyo activo, como el Consorcio Internacional para Combatir los Delitos contra la Vida Silvestre, firmado en San Petersburgo (Rusia) el día 23 de noviembre de 2010, por la Secretaría CITES, la INTERPOL, la Oficina de las Naciones Unidas contra la Droga y el Delito, el Banco Mundial y la Organización Mundial de Aduanas para brindar apoyo coordinado a los organismos encargados de hacer cumplir la ley en materia de flora y fauna silvestres y a las redes subregionales y regionales que promueven día a día la defensa de los recursos naturales.

En la próxima Conferencia de las Partes, que tendrá lugar en Sri Lanka en 2019, se prevé que la senda constructiva e integradora de aplicación del convenio se mantenga, poniendo en valor los esfuerzos de todas las partes en la conservación de las especies en comercio, y se estudien las cuestiones más controvertidas de su aplicación.

\section{Conclusiones}

El comercio de productos derivados de vida silvestre representa un importante valor económico en el contexto del comercio mundial.

En los más de cuarenta años que lleva funcionando, CITES ha demostrado ser una herramienta eficaz para regular el comercio de especies de fauna y flora, favoreciendo el uso sostenible de los recursos naturales y permitiendo un desarrollo económico de las comunidades locales.
Los grandes beneficios que generan los productos de vida silvestre han provocado, en los últimos años, un incremento considerable del comercio ilegal, implicando a redes organizadas de delincuencia a nivel internacional. Este incremento en el tráfico ilegal ha suscitado una gran preocupación internacional y se están abordando importantes medidas para combatirlo.

La importancia de estos aspectos repercute directamente sobre el comercio sostenible de la vida silvestre, satisfaciendo el consumo y la legalidad de los intercambios comerciales y velando por un mercado de vida salvaje amplio, sano y equilibrado, que expresa la voluntad de los Gobiernos por alcanzar el equilibrio entre el desarrollo y la conservación de las especies de fauna y flora silvestres, persiguiendo su objetivo último y aplicando las herramientas necesarias que esbozan un futuro sostenible y proporcionado.

\section{Bibliografía}

[1] CITES (1992). Reconocimiento de las ventajas del comercio de fauna y flora silvestres. Resolución Conf. 8.3 (Rev. CoP13). Kyoto (Japón), 2-13 marzo. Disponible en: http:// www.cites.org/esp/res/all/08/S08-03R13.pdf. (Recuperado: 2017, 29 de julio).

[2] CITES (1997). Aplicación de la Convención a las especies maderables. Resolución Conf. 10.13 (Rev. CoP15). Harare (Zimbabue), 9-20 junio. Disponible en: $h$ ttp://www.cites.org/esp/ res/all/10/S10-13R15.pdf. (Recuperado: 2017, 29 de julio).

[3] CITES (2013). Visión Estratégica: 2008-2020. Resolución Conf. 16.3 (Rev. CoP17). Bangkok (Tailandia), 3-14 Marzo 2013. Disponible en: https://cites.org/sites/default/files/document/ S-Res-16-03-R17.pdf. (Recuperado: 2017, 29 de julio).

[4] CITES (2014). Gestión de cupos de exportación establecidos nacionalmente. Resolución Conf. 14.7 (Rev. CoP15). La Haya (Países Bajos), 3-15 junio 2007. Disponible en: $\triangleright$ 
https://cites.org/sites/default/files/document/ S-Res-14-07-R15.pdf. (Recuperado: 2017, 7 de agosto).

[5] CITES (2014). La dimensión internacional del comercio ilegal de especies silvestres. Disponible en: https://cites.org/esp/ node/15648 (Recuperado: 2017, 2 de agosto).

[6] CITES (2017). Secretaría CITES, homepage (en línea). Disponible en: www.cites.org (Recuperado: 2017, 1 de agosto).

[7] CITES y OMC (2015). La CITES y la OMC: promover la cooperación con miras a un desarroIlo sostenible. Disponible en: https://www.wto. org/spanish/res_s/booksp_s/citesandwto15_s. pdf. (Recuperado: 2017, 3 de agosto).

[8] COMISIÓN EUROPEA (2014). Communication from the Commission to the Council and the European Parliament on the EU Approach against Wildlife Trafficking. Brussels.

[9] HAKEN, J. (2011). Transnational Crime in the Developing World. Disponible en: http://www. gfintegrity.org/report/briefing-paper-transnational-crime. (Recuperado: 2017, 29 de julio).

[10] HUTTON, J. y WEBB, G. (2002). «Legal trade snaps back» pp. 1-10. En: Crocodiles. Proceedings of the 16th Working Meeting of the IUCN-SSC Crocodile Specialist Group. Gland: IUCN.

[11] INSKIPP, T. y WELLS, S. (1979). International Trade in Wildlife. London: Earthscan Publications, IIED.

[12] IUCN (2017). Lista Roja de la UICN. Disponible en: http://www.iucnredlist.org. (Recuperado: 2017, 3 de agosto).

[13] JENKINS, R.W.G. (1987). «The World Conservation Strategy and CITES: principles of the management of crocodilians; pp. 27-31». En: WEBB, G.J.W.; MANOLIS, S.C. y WHITEHEAD, P.J. (eds.). Wildlife Management: Crocodiles and Alligators. Sydney: Surrey Beatty \& Sons.

[14] NACIONES UNIDAS (2015). Lucha contra el tráfico ilícito de fauna y flora silvestres. Resolución 69/314. Disponible en: http://www.un. org/es/comun/docs/?symbol=A/RES/69/314. (Recuperado: 2017, 3 de agosto).
[15] OMC (1947). Acuerdo General sobre Aranceles Aduaneros y Comercio (GATT). Disponible en:https://www.wto.org/spanish/docs_s/ legal_s/gatt47_01_s.htm. (Recuperado: 2017, 4 de agosto).

[16] PNUMA (2005). Manual de Medio Ambiente y Comercio, 2. a ed., Canadá. Programa de las Naciones Unidas para el Medio Ambiente. División de Tecnología, Industria y Economía, Unidad de Economía y Comercio, y el Instituto Internacional para el Desarrollo Sustentable. Disponible en: https://www.unep.org/greeneconomy/sites/unep.org.greeneconomy/files/ publications/handbook_sp.pdf. (Recuperado: 2017, 6 de agosto).

[17] ROSS, J. P. (ed.) (1998). Crocodiles. Status survey and conservation Action Plan. 2. a ed. IUCN/SSC Crocodile Specialist Group. IUCN, Gland, Switzerland \& Cambridge, UK. Disponible en: http://iucncsg.org/ph1/modules/ Publications/action_plan1998/plan1998a.htm. (Recuperado: 2017, 27 de julio).

[18] UNEP-WCMC (2017). Base de datos de especies, homepage (en línea). Disponible en: http://www.unep-wcmc (Recuperado: 2017, 31 de julio).

[19] UNODOC (Oficina de las Naciones Unidas contra la Droga y el Delito) (2017). Wildlife crime worth USD 8-10 billion annually, ranking it alongside human trafficking, arms and drug dealing in terms of profits: UNODC chief. Disponible en: https://www.unodc.org/unodc/ en/frontpage/2014/May/wildlife-crime-worth8-10-billion-annually.html (Recuperado: 2017, 30 de julio).

[20] WALLER, T. y MICUCCI, P. (1995). «Los yacarés en Argentina: hacia un aprovechamiento sustentable» pp. 81-112. En: LARRIERA, A. y VERDADE, L.M.(eds.).Laconservación yelmanejo de caimanes y cocodrilos de América Latina. Santo Tomé: Fundación Banco Bica.

[21] WOODWARD, A. R.; MOORE, C. y DELANEY, M. (1992). Experimental alligator harvest. Final report. Study no. 7567, June 1992. Gainesville, FL. USA: Florida Game and Fresh Water Fish Commission. 
